\title{
An improved hydrometeor detection method for millimeter-wavelength cloud radar
}

\author{
Jinming Ge ${ }^{1}$, Zeen Zhu ${ }^{1}$, Chuang Zheng ${ }^{1}$, Hailing Xie ${ }^{1}$, Tian Zhou ${ }^{1}$, Jianping Huang ${ }^{1}$, and Qiang Fu ${ }^{1,2}$ \\ ${ }^{1}$ Key Laboratory for Semi-Arid Climate Change of the Ministry of Education and College of Atmospheric Sciences, \\ Lanzhou University, Lanzhou, 730000, China \\ ${ }^{2}$ Department of Atmospheric Sciences, University of Washington, Seattle, WA 98105, USA
}

Correspondence to: Qiang Fu (qfu@atmos.washington.edu)

Received: 21 November 2016 - Discussion started: 8 December 2016

Revised: 6 May 2017 - Accepted: 28 June 2017 - Published: 27 July 2017

\begin{abstract}
A modified method with a new noise reduction scheme that can reduce the noise distribution to a narrow range is proposed to distinguish clouds and other hydrometeors from noise and recognize more features with weak signal in cloud radar observations. A spatial filter with central weighting, which is widely used in cloud radar hydrometeor detection algorithms, is also applied in our method to examine radar return for significant levels of signals. "Square clouds" were constructed to test our algorithm and the method used for the US Department of Energy Atmospheric Radiation Measurements Program millimeterwavelength cloud radar. We also applied both the methods to 6 months of cloud radar observations at the Semi-Arid Climate and Environment Observatory of Lanzhou University and compared the results. It was found that our method has significant advantages in reducing the rates of both failed negative and false positive hydrometeor identifications in simulated clouds and recognizing clouds with weak signal from our cloud radar observations.
\end{abstract}

\section{Introduction}

Clouds, which are composed of liquid water droplets, ice crystals or both, cover about two-thirds of the Earth surface at any time (e.g., King et al., 2013). By reflecting solar radiation back to the space (the albedo effect) and trapping thermal radiation emitted by the Earth surface and the lower troposphere (the greenhouse effect), clouds strongly modulate the radiative energy budget in the climate system (e.g., Fu et al., 2002; Huang et al., 2006a, b, 2007; Ramanathan et al., 1989;
Jing Su et al., 2008). Clouds are also a vital component of water cycle by connecting the water-vapor condensation and precipitation. Despite the importance of clouds in the climate system, they are difficult to represent in climate models (e.g., Williams and Webb, 2009), which causes the largest uncertainty in the predictions of climate change by general circulation models (GCMs; e.g., Randall, 2007; Stephens, 2005; Williams and Webb, 2009).

Cloud formation, evolution and distribution are governed by complex physical and dynamical processes on a wide range of scales from synoptic motions to turbulence (Bony et al., 2015). Unfortunately, the processes that occur on smaller spatial scales than a GCM grid box cannot be resolved by current climate models, and the coupling between large-scale fluctuations and cloud microphysical processes is not well understood (e.g., Huang et al., 2006b; Mace et al., 1998; Yan et al., 2015; Yuan et al., 2006). Moreover, the cloud horizontal inhomogeneity and vertical overlap are not resolved by GCMs (Barker, 2000; Barker and Fu, 2000; Fu et al., 2000a, b; Huang et al., 2005; Li et al., 2015). To better understand cloud processes to improve their parameterization in climate models and reveal their evolution in response to climate change, long-term continuous observations of cloud fields in terms of both macro- and microphysical properties are essential (e.g., Ackerman and Stokes, 2003; Sassen and Benson, 2001; Thorsen et al., 2011; Wang and Sassen, 2001).

Millimeter-wavelength cloud radars (MMCRs) can resolve cloud vertical structure for their occurrences and microphysical properties (e.g., Clothiaux et al., 1995; Kollias et al., 2007a; Mace et al., 2001). The wavelengths of MMCRs are shorter than those of weather radars, making them 
sensitive to cloud droplets and ice crystals and able to penetrate multiple cloud layers (e.g., Kollias et al., 2007a). Because of their outstanding advantages for cloud research, millimeter-wavelength radars have been deployed on various research platforms including the first space-borne millimeterwavelength Cloud Profiling Radar (CPR) onboard the CloudSat (Stephens et al., 2002). Ground-based cloud radars are operated at the US Department of Energy's Atmospheric Radiation Program (ARM) observational sites (formerly MMCRs, now replaced with a new generation of Ka-band zenith radar; KAZR; e.g., Ackerman and Stokes, 2003; Clothiaux et al., 1999, 2000; Kollias et al., 2007b; Protat et al., 2011) and in Europe (Illingworth et al., 2007; Protat et al., 2009). In July 2013, a KAZR was deployed in China at the SemiArid Climate and Environment Observatory of Lanzhou University (SACOL) site (latitude of $35.946^{\circ} \mathrm{N}$, longitude of $104.137^{\circ} \mathrm{E}$; altitude of $1.97 \mathrm{~km}$; Huang et al., 2008), providing an opportunity to observe and reveal the detailed structure and process of the midlatitude clouds over the semi-arid regions of East Asia.

Before characterizing the cloud physical properties from the cloud radar return signal, we first need to distinguish and extract the hydrometeor signals from the background noise (i.e., cloud mask). A classical cloud mask method was developed in Clothiaux et al. $(2000,1995)$ by analyzing the strength and significance of returned signals. This method consists of two main steps. First any power in a range gate that is greater than a mean value of noise plus 1 standard deviation is selected as a bin containing potential hydrometer signal. Second, a space-time coherent filter is created to estimate the significance level of the potential hydrometer bin signal to be real. This cloud mask algorithm is operationally used for the ARM MMCRs data analysis and was later adopted to the CPR onboard the CloudSat (Marchand et al., 2008).

It is recognized that by visually examining a cloud radar return image, one can easily tell where the return power is likely to be caused by hydrometeors and where the power is just from noise. This ability of the human eye to extract and analyze information from an image has been broadly studied in image processing and computer vision. A number of mathematical methods for acquiring and processing information from images have been developed, including some novel algorithms for noise reduction and edge detection (Canny, 1986; He et al., 2013; Marr and Hildreth, 1980; Perona and Malik, 1990). In this paper, we propose a modified cloud mask method for cloud radar by noticing that removing noise from signal and identifying cloud boundaries are the essential goals of cloud masking. This method reduces the radar noise while preserving cloud edges by employing the bilateral filtering that is widely used in the image processing (Tomasi and Manduchi, 1998). The power weighting probability method proposed by Marchand et al. (2008) is also adopted in our method to prevent the cloud corners from being removed. It is found that our improved hydrometeor de-
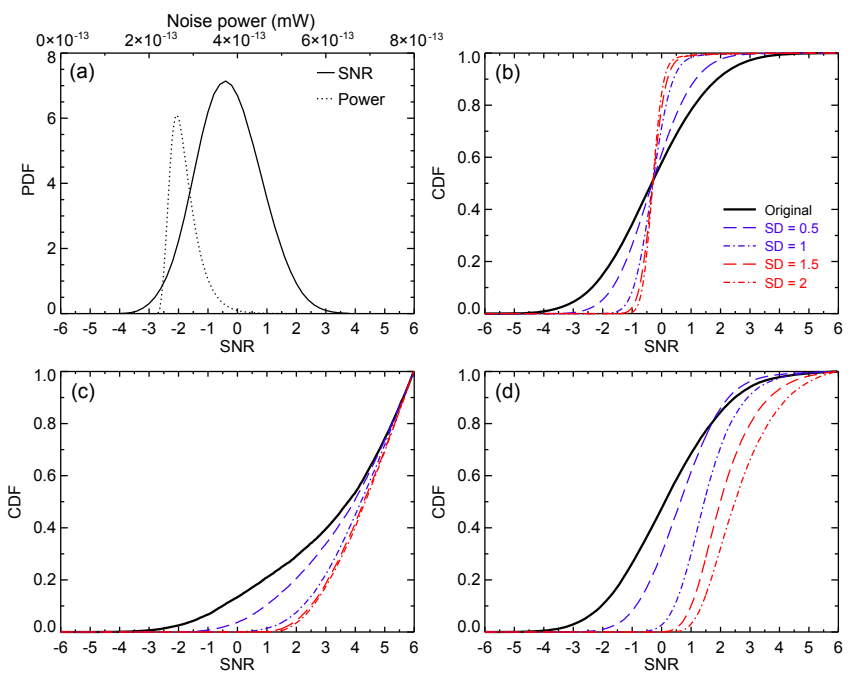

Figure 1. (a) Probability distribution function (PDF) of the noise power and SNR from the KAZR observations on a clear day, 21 January 2014. (b) Cumulative distribution function (CDF) of original and convolved SNR of the noise on the clear day. (c) and (d) CDF of original and convolved SNR of a cloudy case on 4 January 2014 for range gates inside and outside the cloud adjacent to the cloud boundary, respectively. The converted SNR is obtained by using a 2-D Gaussian distribution kernel (Eq. 2).

tection algorithm is efficient in terms of reducing false positives and negatives as well as identifying cloud features with weak signals such as thin cirrus clouds.

The KAZR deployed at the SACOL is described in Sect. 2 and the modified cloud mask algorithm is introduced in Sect. 3. The applications of the new scheme to both hypothetical and observed cloud fields including a comparison with previous schemes are shown in Sect. 4. Summary and conclusions are given in Sect. 5.

\section{The KAZR radar}

The SACOL KAZR, built by ProSensing Inc. of Amherst, MA, is a zenith-pointing cloud radar operating at approximately $35 \mathrm{GHz}$ for the dual-polarization measurements of Doppler spectra. The main purpose of the KAZR is to provide vertical profiles of clouds by measuring the first three Doppler moments: reflectivity, radial Doppler velocity and spectra width. The linear depolarization ratio (LDR; Marr and Hildreth, 1980) can be computed from the ratio of crosspolarized reflectivity to co-polarized reflectivity.

The SACOL KAZR has a transmitter with a peak power of $2.2 \mathrm{kw}$ and two modes working at separate frequencies. One is called "chirp" mode that uses a linear frequencymodulation pulse compression to achieve high radar sensitivity of about $-65 \mathrm{dBZ}$ at $5 \mathrm{~km}$ altitude. The minimum altitude (or range) that can be detected in chirp mode is approximately $1 \mathrm{~km}$ a.g.l. To view clouds below $1 \mathrm{~km}$, a short 
(a)

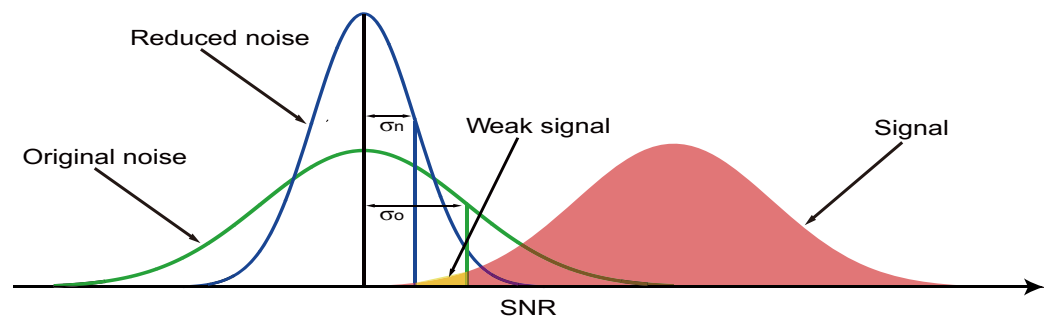

(b)
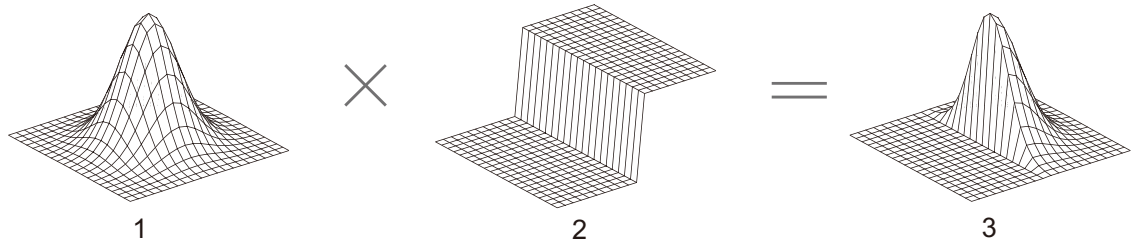

Figure 2. (a) Comparison of original noise, reduced noise and hydrometeor signal distributions. $\sigma_{o}$ and $\sigma_{n}$ are 1 standard deviation of the original and reduced background noise, respectively. (b) Illustration of the bilateral filtering process: (b1) Gaussian kernel distribution in space, (b2) $\delta$ function and (b3) bilateral kernel by combining Gaussian kernel with $\delta$ function.

pulse or "burst mode" pulse is transmitted at a separate frequency just after transmission of the chirp pulse. This burst mode pulse allows clouds as low as $200 \mathrm{~m}$ to be measured. The chirp pulse is transmitted at $34.890 \mathrm{GHz}$ while the burst pulse is transmitted at $34.830 \mathrm{GHz}$. These two waveforms are separated in the receiver and processed separately.

The pulse length is approximately $300 \mathrm{~ns}$ (giving a range resolution of about $45 \mathrm{~m}$ ), while the digital receiver samples the return signal every $30 \mathrm{~m}$. The inter-pulse period is $208.8 \mu$ s, the number of coherent averages is 1 and the number of the fast Fourier transform points is currently set to 512. An unambiguous range is thus $31.29 \mathrm{~km}$, an unambiguous velocity is $10.29 \mathrm{~m} \mathrm{~s}^{-1}$ and a velocity resolution is $0.04 \mathrm{~m} \mathrm{~s}^{-1}$. The signal dwell time is $4.27 \mathrm{~s}$. These operational parameters are set for the purpose of having enough radar sensitivity and accurately acquiring reflectivities of hydrometeors. In this study, we mainly use radar-observed reflectivity (dBZ) data to test our new hydrometeor detection method.

\section{Improved hydrometeor detection algorithm}

The basic assumption in the former cloud mask algorithms (e.g., Clothiaux et al., 1995; Marchand et al., 2008) is that the random noise power follows the normal distribution. Here clear-sky cases in all seasons from the KAZR observations were first analyzed for its background noise power distributions. Figure 1a shows an example of a clear-sky case from 00:00 to 12:00 UTC on 21 January 2014. The noise power is estimated from the top 30 range gates, which includes both internal and external sources (Fukao and Hamazu, 2014). It has an apparent non-Gaussian distribution with a positive skewness of 1.40 (Fig. 1a). The signal-to-noise ratio (SNR) is defined as

$\mathrm{SNR}=10 \log \left(\frac{P_{s}}{P_{n}}\right)$,

where $P_{s}$ is the power received at each range gate in a profile and $P_{\mathrm{n}}$ is the mean noise power that is estimated by averaging the return power in the top 30 range gates, which are between 16.8 and $17.7 \mathrm{~km}$ a.g.l. Since this layer is well above the tropopause, few atmospheric hydrometeors existing in this layer can scatter enough power back to achieve the radar sensitivity. Figure 1a shows that the SNRs for clear skies closely follow a Gaussian distribution. Instead of using radar-received power, the SNR is used as the input in our cloud mask algorithm including estimating the background noise level. This is because in our method the chance of a central range gate being noise or a potential feature relies on the probability of a given range of SNR values following the Gaussian distribution. Note that the mean value of the SNR for the noise power is not zero, but a small negative value of about -0.3 . This is because the mean of the noise power is larger than its the median due to its positive skewed distribution. It is further noted that, for the noise, the distribution of SNR and its mean for the top 30 range gates are the same as those from the lower atmosphere.

The SNR value is treated as the brightness of a pixel in an image $f(x, y)$ in our hydrometeor detection method. In image processing, the random noise can be smoothed out by using a low-pass filter, which gives a new value for a pixel of an image by averaging with neighboring pixels (Tomasi and Manduchi, 1998). The cloud signals are highly correlated in both space and time and have more similar values in near pixels while the random noise values are not corre- 


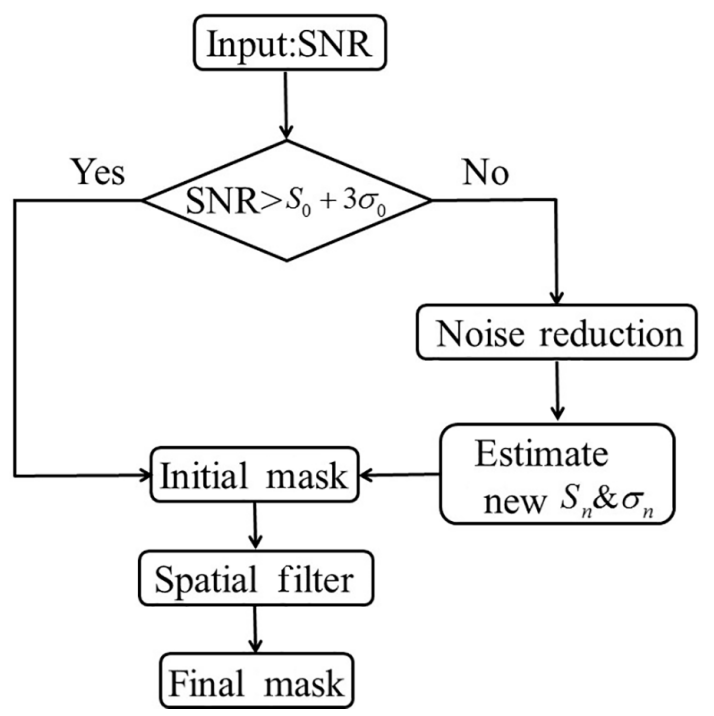

Figure 3. Schematic flow diagram for hydrometeor detection method. $S_{O}$ and $S_{\mathrm{n}}$ are the mean SNR for the original and reduced noise, respectively.

lated. Figure 2a shows a schematic comparison of the original noise, reduced noise and hydrometeor signal distributions: the low-pass filter could efficiently reduce the original radar noise represented by the green line to a narrow bandwidth (blue line) while keeping the signal preserved. By reducing the standard deviations of noise, which shrinks the overlap region of signal and noise and enhances their contrast, the weak signals (yellow area) that cannot be detected based on original noise level may become distinguished.

Following this idea, we develop a non-iterative hydrometeor detection algorithm by applying a noise reduction and a central-pixel weighting schemes. Figure 3 shows the schematic flow diagram of our method. For given mean SNR values $\left(S_{o}\right)$ and 1 standard deviation $\left(\sigma_{o}\right)$ of the original background noise, the input SNR data set is first separated into two groups. The group with values greater than $S_{o}+3 \sigma_{o}$ is considered to be the cloud features that can be confidently identified. Another group with values between $S_{o}$ and $S_{o}+3 \sigma_{o}$ may potentially contain moderate $\left(S_{o}+\sigma_{o}<\mathrm{SNR} \leq\right.$ $\left.S_{o}+3 \sigma_{o}\right)$ to weak $\left(S_{o}<\mathrm{SNR} \leq S_{o}+\sigma_{o}\right)$ cloud signals, which will further go through a noise reduction process. Here $S_{o}$ and $\sigma_{o}$ are estimated from the top 30 range gates of each five successive profiles.

The noise reduction process is performed by convolving radar SNR time-height data with a low-pass filter. The Gaussian filter, which outputs a "weighted average" of each pixel and its neighborhood with the average weighted more towards the value of the central pixel $\left(v_{0}\right)$, is one of the most common functions of the noise reduction filter. A 2-D Gaussian distribution kernel, shown in Fig. 2 $b_{1}$, can be expressed as

$G(i, j)=\frac{1}{2 \pi \sigma^{2}} \exp \left(-\frac{i^{2}+j^{2}}{2 \sigma^{2}}\right)$,

where $i$ and $j$ are the indexes in a filter window and are 0 for the central pixel, and $\sigma$ is the standard deviation of the Gaussian distribution for the window size of the kernel. Equation (2) is used in our study to filter the radar SNR image. Note that the convolution kernel is truncated at about 3 standard deviations away from the mean in order to accurately represent the Gaussian distribution. Figure $1 \mathrm{~b}$ is the cumulative distribution functions of clear-sky SNR by convolving the same data in Fig. 1a with filters that have different kernel sizes $(3 \times 3,5 \times 5,7 \times 7$ and $9 \times 9$ pixels), corresponding to the $\sigma$ ranging from 0.5 to 2 . The original SNR values are distributed from about -5 to 5 . After convolving the image with the Gaussian filter, the SNR distribution can be constrained to a much narrower range. It is clear that the filter with a larger kernel size is more effective in suppressing the noise. Shown in Fig. 1c are results for a cloudy case on 4 January 2014 by applying the filter to the range gates inside the cloud but adjacent to the boundary. It is shown that a larger kernel size shifts the SNR farther away from the noise region. It therefore appears that increasing the standard deviation (i.e., the window size) would reduce the noise and enhance the contrast between signal and noise more effectively. At the same time, however, a larger kernel can also attenuate or blur the high-frequency components of an image (e.g., the boundary of clouds) more. As shown in Fig. 1d, when the window size is increased from $3 \times 3(\sigma=0.5)$ to $9 \times 9(\sigma=2)$, the SNR distribution of the range gates that are outside the cloud but adjacent to the boundary gradually move toward larger values. This will consequently raise the risk of misidentifying cloud boundaries. To solve this problem, a bilateral filtering idea proposed by Tomasi and Manduchi (1998) is adopted here. Considering a sharp edge between cloudy and clear region as shown in Fig. 2b2, we define a $\delta(i, j)$ function that, when the central pixel is on the cloudy or clear side, gives a weighting of 1 to the similar neighboring pixels (i.e., on the same side) and 0 to the other side. After combining this $\delta$ function to the Gaussian kernel in Fig. 2b1, we can get a new nonlinear function called bilateral kernel as shown in Fig. 2b3. It can be written as

$B(i, j)=\frac{1}{2 \pi \sigma^{2}} \exp \left(-\frac{i^{2}+j^{2}}{2 \sigma^{2}}\right) \cdot \delta(i, j)$.

Thus the bilateral kernel will reduce averaging noises with signals, and vice versa. The noise-reduced image $h(x, y)$ is produced by convolving the bilateral kernel with the original input image $f(x, y)$ as

$$
h(x, y)=k^{-1}(x, y) \sum_{j=-w}^{j=w} \sum_{i=-w}^{i=w} f(x+i, y+j) \cdot B(i, j),
$$


where $\pm w$ is the bounds of the finite filter window, and $k^{-1}(x, y)$ is defined as $1 / \sum_{j=-w}^{j=w} \sum_{i=-w}^{i=w} B(i, j)$, which is used to normalize the weighting. Since the bilateral kernel function only averages the central pixel with neighbors on the same side (Fig. 2b), ideally it will preserve sharp edges of a target. We will discuss how to construct the $\delta$ function in order to group the central pixel with its neighbors later in this section. In the noise reduction process, a $5 \times 5$ window size (i.e., 25 bins in total) is specified for the low-pass filter, which is empirically determined by visually comparing the cloud masks with original images. We should keep in mind that a small window size is less effective in noise reduction but a large window is not suitable for recognizing weak signals.

For performing the noise reduction with Eq. (4) in a $5 \times 5$ filter window, the number of range bins $\left(N_{s}\right)$ with signal greater than $S_{o}+3 \sigma_{o}$ are first counted. These $N_{s}$ range bins are then subtracted from the total 25 of the range bins in the filter window. Note that a noise reduction is only applied when the central pixel is among the $25-N_{s}$ bins, and the $\delta$ function is set to be zero for the $N_{s}$ range bins. If the remaining $25-N_{s}$ range bins are all noises, the range bin number $\left(N_{m}\right)$ with SNR greater than $S_{o}+\sigma_{o}$ should be about equal to an integral number $\left(N_{t}\right)$ of $0.16 \times\left(25-N_{s}\right)$ where 0.16 is the probability for a remaining range bin to have a value greater than $S_{o}+\sigma_{o}$ for a Gaussian noise. Thus when $N_{m}$ is equal to or smaller than $N_{t}$, all the $25-N_{s}$ range bins could only contain pure noise and/or some weak cloud signals. In this case, the $\delta$ function is set to 1 for all the $25-N_{s}$ bins. When $N_{m}$ is found to be larger than $N_{t}$, the $25-N_{s}$ range bins might contain a combination of moderate signal, noise and/or some weak clouds. In this case, $S_{o}+\sigma_{o}$ is selected as a threshold to determine whether the pixels are on the same side of the central pixel. If the central pixel has a value greater than $S_{o}+\sigma_{o}$, the $\delta$ function is assigned to 1 for the $25-N_{s}$ pixels with $\mathrm{SNR} \geq S_{o}+\sigma_{o}$, but 0 for the bins with SNR $<S_{o}+\sigma_{o}$. If the central pixel is less than $S_{o}+\sigma_{o}$, the $\delta$ function is assigned to 1 for the pixels with SNR $<S_{o}+\sigma_{o}$, but 0 for the $25-N_{s}$ bins with $\mathrm{SNR} \geq S_{o}+\sigma_{o}$.

After picking out the strong return signals and applying the noise reduction scheme, the new background noise $S_{n}$ and its standard deviation $\sigma_{n}$ are estimated. While $S_{n}$ is the same as $S_{o}$, the $\sigma_{n}$ is significantly reduced, which is a half of $\sigma_{o}$. This will make it possible to identify more hydrometeors as exhibited in Fig. 2a. We assign different confidence level values (which is called the mask value in this study) to the following initial cloud mask according to the SNR; 40 is first assigned to the mask of any range bins with $\mathrm{SNR}>S_{o}+3 \sigma_{o}$ in the original input data. For the rest of the range bins, after applying the noise reduction, if the $\mathrm{SNR}>S_{n}+3 \sigma_{n}$, the mask is assigned a value of 30; if $S_{n}+2 \sigma_{n}<\mathrm{SNR} \leq S_{n}+3 \sigma_{n}$, the mask is 20 ; if $S_{n}+\sigma_{n}<\mathrm{SNR} \leq S_{n}+2 \sigma_{n}$, the mask is 10 ; and the remaining range bin mask is assigned a value of 0 . Thus, a (a) Input SNR
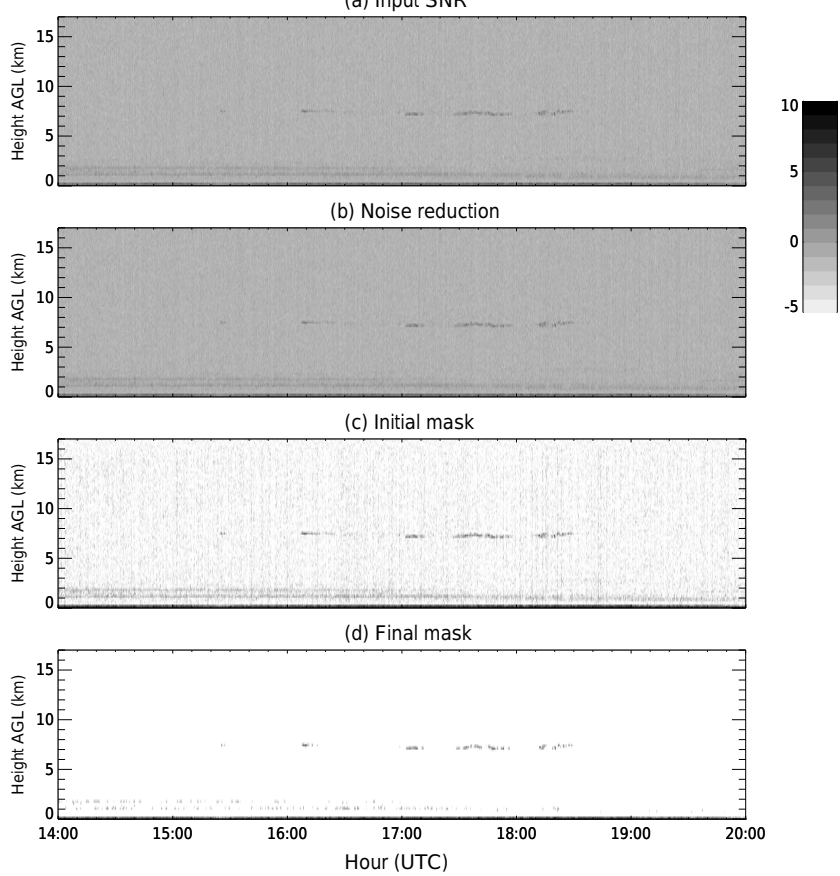

Figure 4. Illustration of the steps of the detection method using the real data from 8 January 2014.

mask value assigned to a pixel represents the confident level for the pixel to be a feature.

To reduce both false positives (i.e., false detections) and false negatives (i.e., failed detections), the next step is to estimate whether a range gate contains significant hydrometeor. Following Clothiaux et al. $(2000,1995)$ and Marchand et al. (2008), a $5 \times 5$ spatial filter is used to calculate the probability of clouds and noise occurring in the 25 range gates. The probability of central-pixel weighting scheme proposed by Marchand et al. (2008) is adopted here, and the weighting for the central pixel is assigned according to its initial mask value. The probability is calculated by

$p=G(L)\left(0.16^{N_{T}}\right)\left(0.84^{N_{0}}\right)$,

where $N_{0}$ is the number of masks with zero mask value, $N_{T}$ is the number of masks with non-zero mask value and $N_{0}+N_{T}=25 ; G(L)$ is the weighting probability of the central pixel that could be a false detection at a given significant level of $L$ (i.e., mask value) in the initial cloud mask. Here $G(0)=0.84, G(10)=0.16, G(20)=0.028$ and $G(\geq$ $30)=0.002$. If $p$ estimated from Eq. (5) is less than a given threshold ( $\left.p_{\text {thresh }}\right)$, then the central pixel is likely to be a hydrometeor signal. The cloud mask value will be set to the same value as in the initial mask if it is non-zero; otherwise it will be set to 10. Likewise, if $p>p_{\text {thresh }}$, then the central pixel is likely to be noise and the mask value will be set to 0 . This process is iterated five times for each pixel to obtain the final cloud mask. 

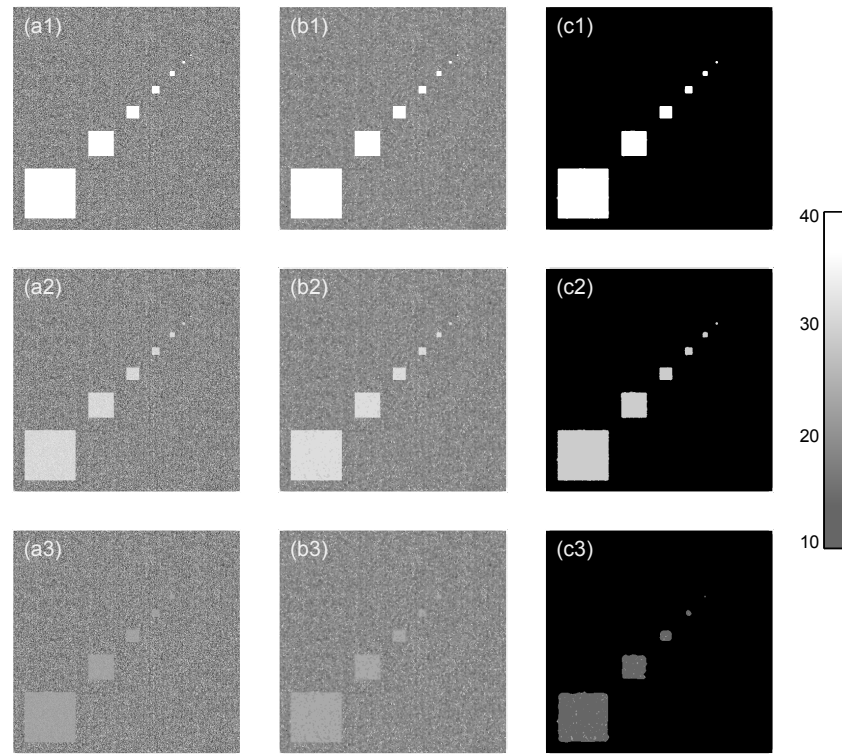

Figure 5. Panels (a1), (a2) and (a3) are three "square clouds" that have strong, moderate and weak SNR values with random Gaussian noise used to test the detection method. Panels (b1), (b2) and (b3) are SNR distributions after convolving the data with a bilateral kernel. Panels (c1), (c2) and (c3) are the final cloud mask filtered by the spatial filter.

Following Marchand et al. (2008), who explained the logic of choosing a proper threshold, $p_{\text {thresh }}$ is calculated as

$p_{\text {thresh }}=\left(0.16^{N_{\text {thresh }}}\right)\left(0.84^{25-N_{\text {thresh }}}\right)$.

Note that a smaller $p_{\text {thresh }}$ will keep the false positives lower but increase the false negative. Herein we adopt the $p_{\text {thresh }}$ of $5.0 \times 10^{-12}$ used in Clothiaux et al. (2000), which is approximately equivalent to $N_{\text {thresh }}=13$.

Figure 4 illustrates the main steps of our detection method by using the data from 8 January 2014. Figure 4a is the original SNR input. Figure 4b shows the SNR distribution after the noise reduction process. One can see that the SNR, after being compressed to a narrow range, becomes much smoother than original input. This step significantly increases the contrast between signal and noise. Figure $4 \mathrm{c}$ indicates the range gates that potentially contain hydrometeors in the initial cloud mask. Figure $4 \mathrm{~d}$ is the final result after applying the spatial filter.

\section{Results}

\subsection{Detection test}

To test the performance of our hydrometeor detection method, we create seven squares of SNR with sides of 100, $50,25,15,10$ and 5 and three bins to mimic the radar "timeheight" observations as shown in Fig. 5. The background
Table 1. Summary of false positives and failed negatives for hypothetical strong, moderate and weak cloud cases in Fig. 5a1, a2 and $\mathrm{a} 3$, respectively.

\begin{tabular}{lllrrr}
\hline & & \multicolumn{3}{c}{ Cloud mask confidence level } \\
\cline { 3 - 6 } Cloud & Performance & $\geq 10$ & $\geq 20$ & $\geq 30$ & $\geq 40$ \\
type & $(\%)$ & & & & \\
\hline \multirow{2}{*}{ Strong } & False positive & 0.048 & 0.044 & 0.009 & 0 \\
& Failed negative & 0.244 & 0.244 & 0.244 & 0.244 \\
Moderate & False positive & 0.103 & 0.103 & 0.063 & 0 \\
& Failed negative & 0.229 & 0.229 & 0.229 & 100 \\
Weak & False positive & 0.007 & 0.006 & 0.003 & 0 \\
& Failed negative & 9.774 & 96.788 & 100 & 100 \\
\hline
\end{tabular}

noise is randomly given by a Gaussian distribution with a mean $S_{0}$ and a standard deviation $\sigma_{0}$. The targets in panels a1, a2 and a3 are set with different SNR values to represent situations in which clouds have strong, moderate and weak signals, respectively. In panel a1 the target signals are set to be $S_{0}+10 \sigma_{0}$. In panel a2, the target signals distribute from $S_{0}+\sigma_{0}$ to $S_{0}+3 \sigma_{0}$ with a mean value of $S_{0}+2 \sigma_{0}$. In panel a3, the target SNRs range from $S_{0}$ to $S_{0}+\sigma_{0}$ with a mean value of $S_{0}+0.5 \sigma_{0}$.

The three middle panels in Fig. 5 show the results after applying the noise reduction. Again, comparing with the input signals, we can see that the background noise is well compressed and becomes smoother. The shapes of the square targets are all well maintained with sharp boundaries for strong and moderate signals (see Fig. 5b1 and b2). In Fig. $5 \mathrm{~b} 3$ for weak signals, the three-bin square target is not obvious while the other six squares are still distinguishable. To separate the compressed background noise from hydrometeor signals, the $5 \times 5$ spatial filter is further applied to the noise-reduced data. The three right panels in Fig. 5 show the final mask results. Generally, the hydrometeor detection method can identify those targets well. Six of the seven square targets can be identified for clouds with strong and moderate SNR. The $3 \times 3$ square is missed because the small targets cannot be resolved by the $5 \times 5$ spatial filter. Since the temporal resolution of KAZR is about $4 \mathrm{~s}$, we expect that a cloud only having three bins in horizontal would be rare. For the targets with weak SNR values, the $3 \times 3$ and $5 \times 5$ square targets are missed, but the rest five square targets are successfully distinguished and their boundaries are well maintained as shown in Fig. $5 \mathrm{c} 3$.

To further demonstrate the performance of our method for detecting the hypothetical clouds in Fig. 5a1, a 2 and a3, the false and failed detection rates are listed in the Table 1. For strong signals, no background noise pixel is misidentified as one containing hydrometeors at level 40. Although at levels less than 40, some noise pixels around the edges of targets are identified as signals, the false detection is within $0.05 \%$. The failed detection rate is about $0.24 \%$. For moderate signals, the failed detection rate is still as small as $0.23 \%$, while the 

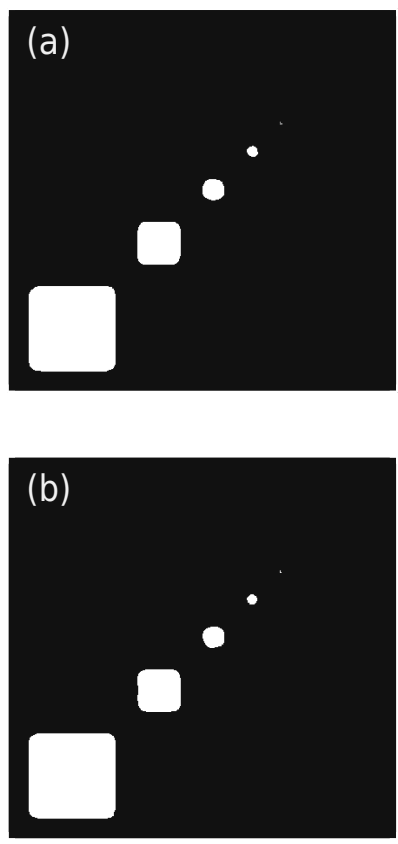

and 6 , it is obvious that our hydrometeor detection method can maintain the cloud boundary well, keep both false and failed detection rate as low as a few percent for strong and moderate cloud cases and has a remarkable advantage in recognizing weak signals.

It is noted that the ARM program has recently developed a new operational cloud mask algorithm for the KAZRs by applying the Hildebrand and Sekhon (1974) technique to determine the SNR values along with the spatial filter (Johnson, $\mathrm{K}$., personal communication, 2017). It is our future research task to compare our algorithm with the ARM's new operational algorithm.

\subsection{Application to the SACOL KAZR observations}

Our hydrometeor detection method was then applied to the winter and summer (December 2013 and January, February, June, July and August 2014) KAZR data at the SACOL. A micropulse lidar (MPL) transmitted at $527 \mathrm{~nm}$ is operated near the KAZR. Lidar is more sensitive to thin cirrus clouds and thus used to assess the performance of our algorithm. Figure $7 \mathrm{a}, \mathrm{b}$ and $\mathrm{c}$ show a 1-day example of radar reflectivity, normalized backscatter and depolarization ratio of lidar, respectively. The cloud masks from our detection method and the ARM MMCR method are shown in Fig. $7 d$ and e. The MPL feature mask is derived by modifying the method developed in Thorsen et al. (2015) and Thorsen and Fu (2015; see Fig. 7f). The vertical and horizontal resolutions of the radar and lidar are different, and we map the observed data and derived feature mask on the same height and time coordinates for the purpose of a comparison. A distinct thin feature layer appears at about $8 \mathrm{~km}$ from 15:00 to 18:30 UTC during the lidar observation, which is clearly identified as a cirrus cloud using the depolarization ratio. The contrast between the cirrus layer and background from the KAZR observation (Fig. 7a) is very weak, and only a few range gates are identified as the hydrometeors using the method without the noise reduction and weighting (Fig. 7d). However, our cloud mask method can find more range gates (about 2.8 times of ARM's result). All these increased range bins from our method are also detected as thin cirrus by the MPL (Fig. 7f). Another apparent discrepancy exists in the low atmosphere layer. A non-negligible number of range gates at about $2 \mathrm{~km}$ are recognized as hydrometeor echoes by our method but mostly missed by former technique. This feature layer is also apparent in lidar observations with both relative large backscatter intensities and depolarization ratios (Fig. 7b and c). MPL recognizes this feature as an aerosol layer. From our KAZR observations, we did find some dust events that were detected by this millimeter-wavelength radar (see the auxiliary Fig. 1). Those feature echoes detected by our method might be partly caused by large dust particles. Although the dust is not desired for cloud mask, the appearance of those particles does prove the ability of our method to recognize weak signals. 

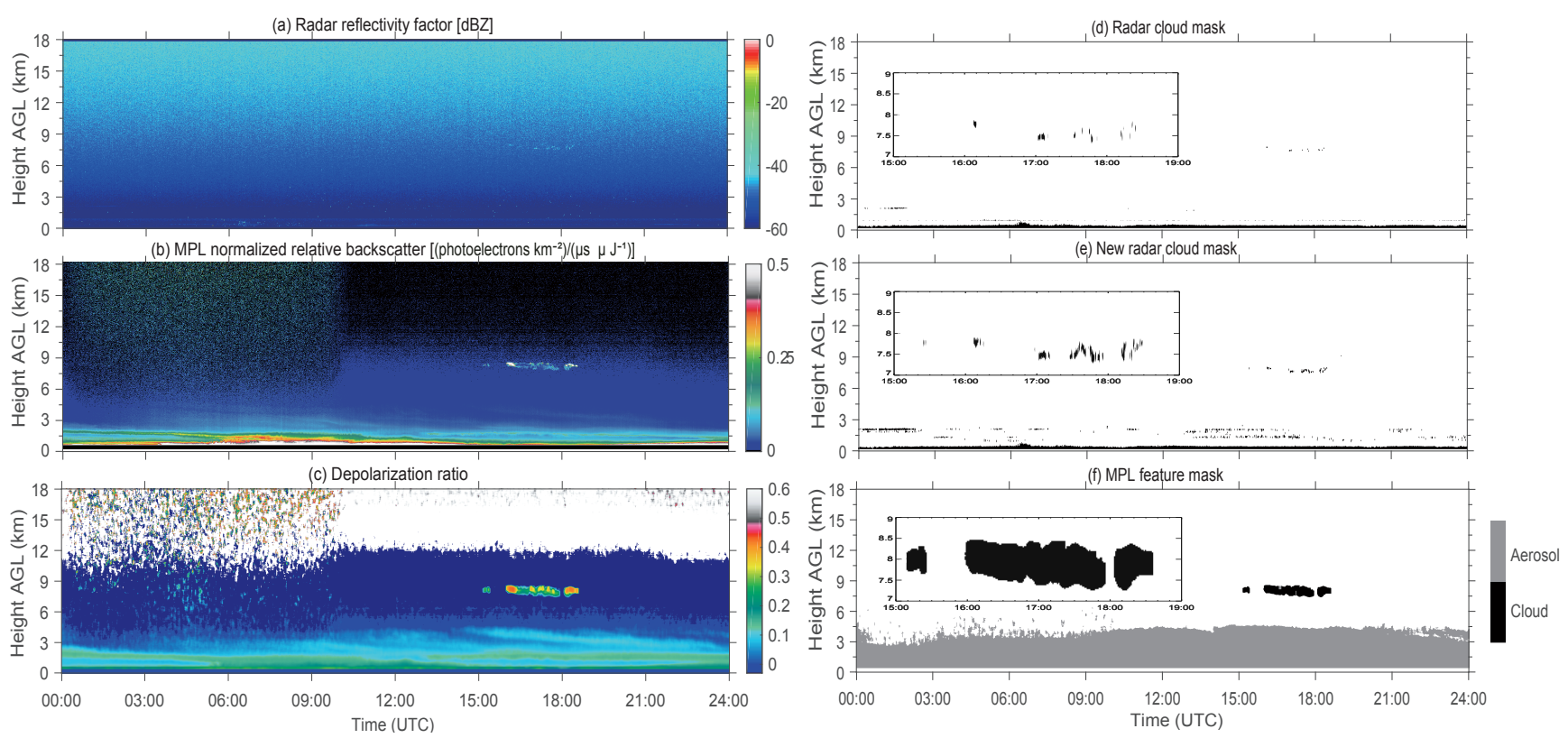

Figure 7. One-day example of radar- and lidar-observed cirrus clouds at the SACOL on 8 January 2014. (a) KAZR reflectivity; (b) MPL normalized backscatter intensity; (c) MPL depolarization ratio; (d) radar cloud mask derived by the ARM MMCR algorithm; (e) radar cloud mask derived by our new method; (f) MPL feature mask. Three windows in (d), (e) and (f) show the zoom-in views of cirrus masks.
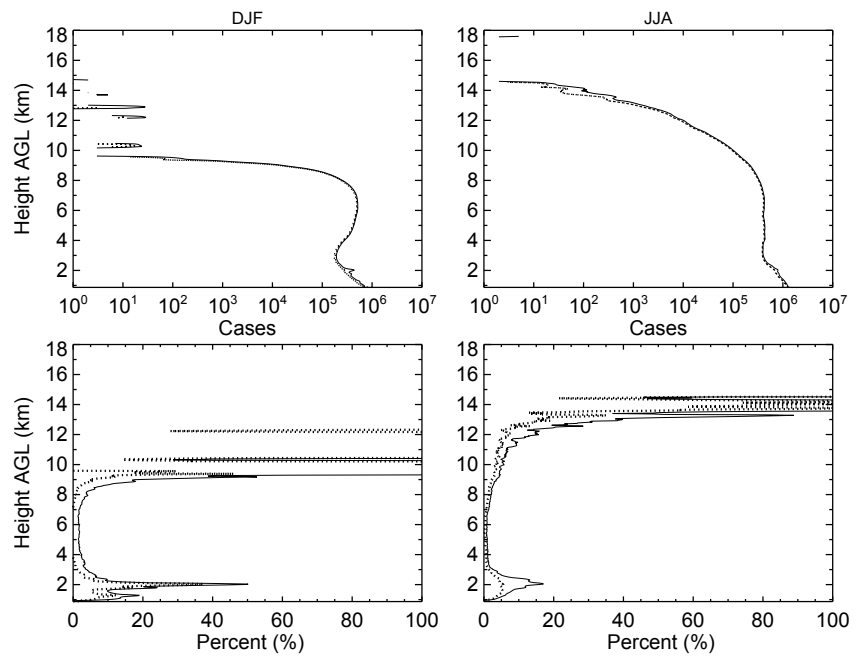

Figure 8. The upper panel shows the number of occurrences of the detected hydrometeor range bins from the two methods. The solid line is the number of range gates derived from our method. The dotted line from the ARM MMCR algorithm. The lower two panels demonstrate the increased percentage of hydrometeor bins from our method comparing to the ARM MMCR algorithm. The solid line is calculated by applying both noise reduction and central-pixel weighting schemes, while the dashed line is calculated by only applying the central-pixel weighting scheme in our detection method.

The upper two panels in Fig. 8 compare the number of occurrences of the detected hydrometeor range bins from our methods with that from the ARM MMCR algorithm for the
Table 2. Mean values of four quantities for increased KAZR feature and noise pixels.

\begin{tabular}{lrr}
\hline & $\begin{array}{r}\text { Increased KAZR } \\
\text { feature }\end{array}$ & $\begin{array}{r}\text { KAZR } \\
\text { noise }\end{array}$ \\
\hline MPL backscatter & 0.15 & 0.10 \\
MPL depolarization ratio & 0.16 & 0.12 \\
KAZR SNR & 3.9 & 0.1 \\
KAZR LDR & -3.0 & -0.4 \\
\hline
\end{tabular}

Table 3. Confusion matrix of KAZR mask results from our method and the ARM MMCR algorithm estimated by MPL observations.

\begin{tabular}{lrr}
\hline & Our method & MMCR method \\
\hline True positive & $70.7 \%$ & $68.9 \%$ \\
True negative & $95.4 \%$ & $95.5 \%$ \\
False positive & $4.6 \%$ & $4.5 \%$ \\
False negative & $29.3 \%$ & $31.1 \%$ \\
\hline
\end{tabular}

6 months of data. Generally, one can see that the variations of the identified hydrometeor numbers with height from the two techniques are in a good agreement. The distinct discrepancies appear at about $2 \mathrm{~km}$ in winter and above $13 \mathrm{~km}$ in summer, when our method apparently identifies more hydrometeors. To quantitatively evaluate the two schemes used in our algorithm and illustrate the improvements of our method, we plot the percent change of the increased hydrometeors from our method by comparing it to the ARM MMCR method in 

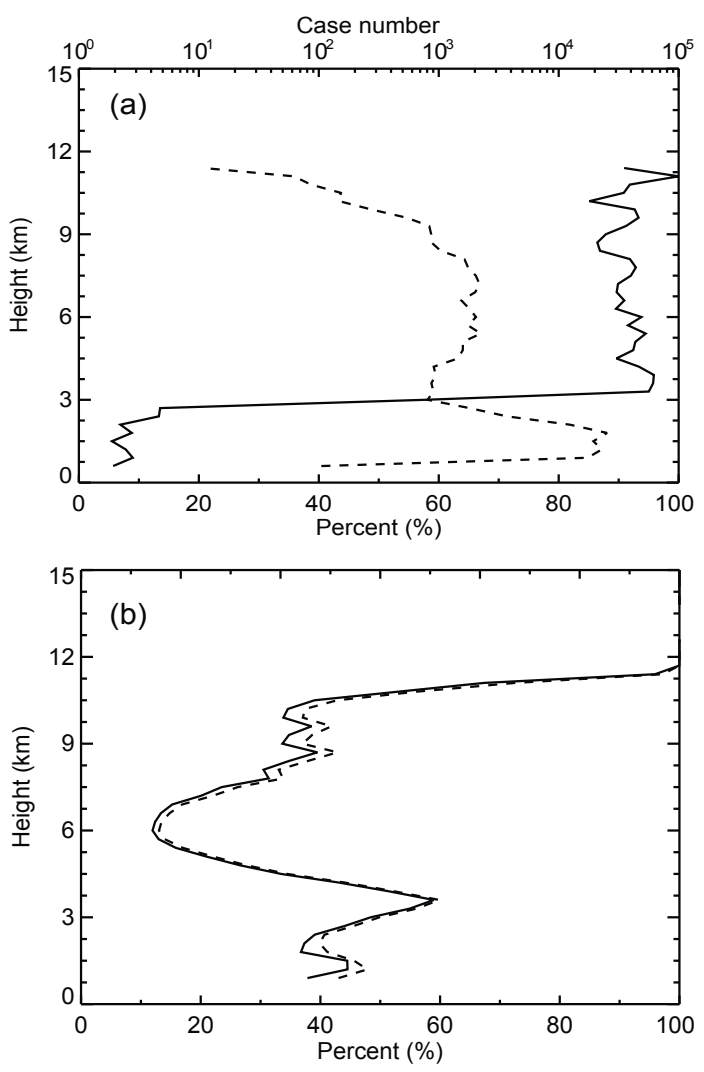

Figure 9. (a) A comparison of the increased detections with the MPL observations. (b) The percentage of the cloud pixels identified by MPL but not by KAZR in the total MPL detected cloud pixels. The solid line in Fig. 9a is the percentage of increased detections seen by both KAZR with our method and MPL as compared with the total increased detections. The dash line in Fig. 9a is the number of increased detections. The solid lines in Fig. $9 \mathrm{~b}$ represents for the algorithm with noise reduction step. The dash line in Fig. $9 \mathrm{~b}$ is for the method without noise reduction scheme.

the lower two panels in Fig. 8. As expected from the results in the test square clouds, our method can identify more signals. The remarkable feature is that the increased percentage is over $20 \%$ at high altitude, indicating that our method can recognize more cirrus clouds. The increased percentage of hydrometeor derived only with the weighting scheme (dashed line) and with both the noise reduction and weighting schemes (solid line) varies differently with height to demonstrate the individual contribution of the scheme to the improvement of our method. In winter, the number of the detected hydrometeors with only the weighting scheme is almost the same as that from the ARM method at layer from 3.5 to $9 \mathrm{~km}$ a.g.l., while this number will increase by about $5 \%$ if the noise reduction scheme is involved, indicating that some hydrometeors with weak SNR values may exit in this layer. Above and below this atmospheric layer, the increased percentage is largely determined by the weighting scheme. In summer, the two lines almost overlap each other between
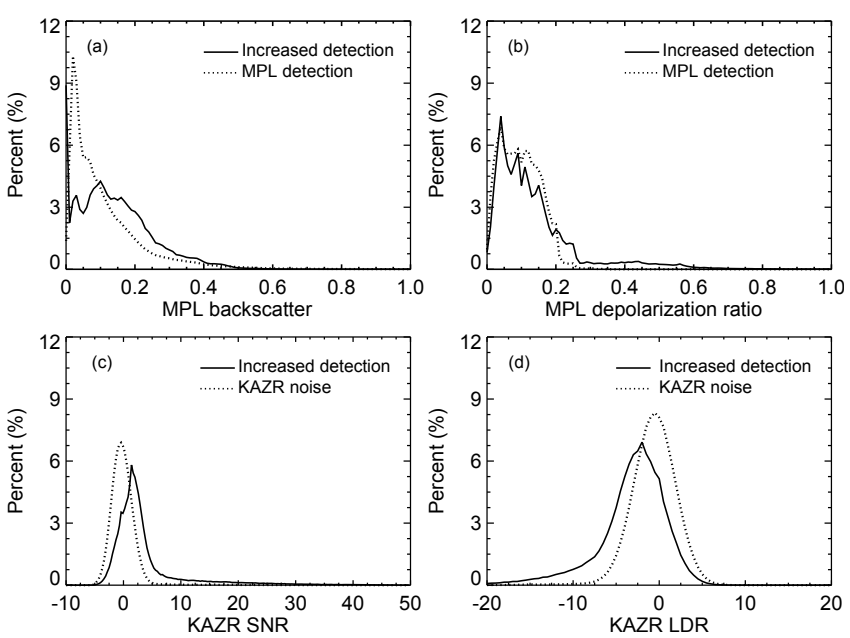

Figure 10. PDF of (a) MPL backscatter, (b) MPL depolarization ratio, (c) KAZR SNR and (d) KAZR LDR for the increased KAZR detections (solid line) and KAZR noise (dashed line) pixels.

3.5 and $9.5 \mathrm{~km}$ with values below $5 \%$, revealing that the bins found by our method in the mid-atmospheric layer are mainly around the boundaries of clouds. We may infer that in summer season, clouds in the middle level are usually composed of large droplets with strong SNR values. The two lines are gradually moving apart with height. This is because hydrometeors in the upper troposphere usually have smaller size that cause weak SNR values, which will be effectively detected by the noise reduction scheme.

We also analyzed the data when both KAZR and MPL observations are available and compared our KAZR cloud mask with MPL feature detection. Figure 9a shows the percentage of the increased detections identified by both KAZR with our method and MPL observations as normalized to the KAZR total increased detections. Here we should point out that MPL has difficulty distinguishing dust from clouds (especially cirrus clouds). Unfortunately, there exists a large amount of dust aerosols over the SACOL region. We visually examined several cases and found that many MPL signals, which should be clouds, are misidentified as aerosols. For this reason, we compare the increased KAZR detections with the features (i.e., cloud and aerosol) detected by MPL above $3 \mathrm{~km}$. It is obvious that more than $90 \%$ of increased detections are also detected as features by MPL. Below $3 \mathrm{~km}$, we calculated the percentage by comparing the KAZR detections only with the cloud pixels detected by MPL since aerosol is always present in the lowest several kilometers. To test whether those increased detections that are not identified as cloud by MPL under $3 \mathrm{~km}$ are signal or noise, we examined the probability distribution functions (PDFs) of MPL normalized aerosol backscatter and depolarization corresponding to the increased KAZR feature and KAZR noise regions in Fig. 10a and b. The PDFs of MPL backscatter for the KAZR feature and noise regions are quite different 
(Fig. 10a), with mean backscatter of 0.15 for feature and 0.10 (photoelectrons $\left.\mathrm{km}^{-2}\right) /\left(\mu \mathrm{s} \mu \mathrm{J}^{-1}\right.$ ) for noise. The mean of the MPL depolarization ratio is 0.16 for feature and 0.12 for noise although the PDFs are similar (Fig. 10b), because dust is the main aerosol type over this region. We also plot the PDFs of KAZR SNR and LDR for the increased feature and noise pixels (Fig. 10c and d). The PDFs of SNR and LDR are Gaussian-like for noise pixels and are quite different from those for the increased detections. Table 2 shows the mean values of the four quantities shown in Fig. 10. All the differences of these mean values between KAZR noise and increased feature regions pass the significant test at $95 \%$ confidence level except for the MPL depolarization ratio. These increased features from our feature mask could thus be dust (and/or some plankton) but cannot be the false positive. Figure $9 \mathrm{~b}$ shows the profile of the false negative (i.e., the percentage of the cloud pixels identified by MPL but not by KAZR in the total MPL-detected cloud pixels). We can see that our method with the noise reduction has relative smaller false negatives especially in the layers under $3 \mathrm{~km}$ and between 7 and $10 \mathrm{~km}$. Table 3 is the confusion matrix of the KAZR feature mask results from both our and the ARM MMCR methods estimated by MPL cloud feature. Overall, $70.7 \%$ of the cloud mask identified by MPL was also recognized by the new method, while this percent is $68.9 \%$ for the algorithm without noise reduction. The difference of false positive between the two methods is only $0.1 \%$ as shown in Table 3. These numbers show an improvement of our method of recognizing weak signals by comparing with the results from the ARM MMCR method; however, they cannot be used to assess the accuracy of our method due to the issue of MPL feature detection.

\section{Summary and discussion}

Based on image noise reduction technique, we propose a modified method to detect hydrometeors from cloud radar return signals. The basic idea is to treat the SNR value of each range gate as a pixel brightness and suppress the SNR distributions of noise to a narrow range by convolving with a 2-D bilateral kernel which can effectively avoid blurring the highfrequency components (i.e., boundaries of a target). After the noise smoothing process, a special filter with a central-pixel weighting scheme is used to obtain the final cloud mask. The detection of the test square clouds shows that there are two remarkable advantages of our method. First, the noise reduction scheme of our algorithm can enhance the contrast between signal and noise, while keeping the cloud boundaries preserved and detecting more hydrometeors with weak SNR values. Second, both false positive and failed negative rates for strong and moderate clouds can be reduced to acceptably small values. A comparison of radar and lidar observations further highlight the advantage of our method for recognizing weak cloud signal in application. 
Appendix A

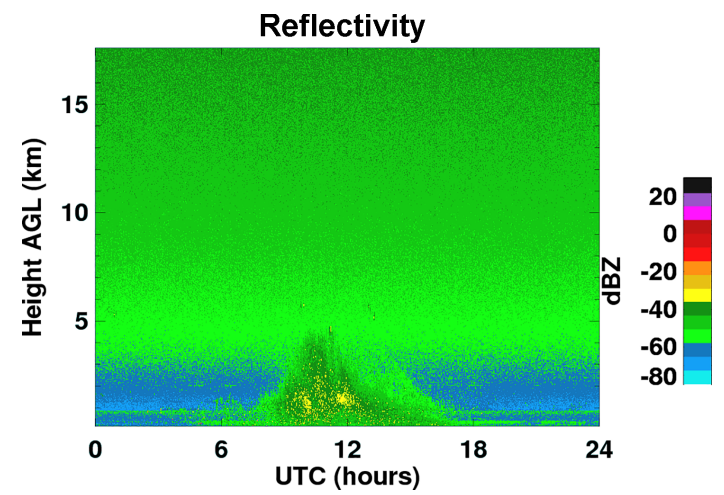

Figure A1. KAZR reflectivity on 29 January 2014 at the SACOL, indicating a dust event. The morphology and power level of the return signal are not apparent for a cloud from the surface to the height of $5 \mathrm{~km}$ between 08:00 and 16:00 UTC. 
Data availability. The datasets used in this paper are available by contacting the author at gejm@1zu.edu.cn.

Competing interests. The authors declare that they have no conflict of interest.

Acknowledgements. This work was supported by the National Science Foundation of China (41430425, 41575016, 41521004, 41505011), China 111 project (no. B13045) and the Fundamental Research Funds for the Central University (lzujbky-2016-k01).

Edited by: Xiaohong Liu

Reviewed by: three anonymous referees

\section{References}

Ackerman, T. P. and Stokes, G. M.: The Atmospheric Radiation Measurement program, Phys. Today, 56, 14-14, 2003.

Barker, H. W.: Indirect aerosol forcing by homogeneous and inhomogeneous clouds, J. Climate, 13 , 4042-4049, https://doi.org/10.1175/15200442(2000)013<4042:iafbha>2.0.co;2, 2010.

Barker, H. W. and Fu, Q.: Assessment and optimization of the gamma-weighted two-stream approximation, J. Atmos. Sci., 57, 1181-1188, https://doi.org/10.1175/15200469(2000)057<1181:aaootg>2.0.co;2, 2000.

Bony, S., Stevens, B., Frierson, D. M. W., Jakob, C., Kageyama, M., Pincus, R., Shepherd, T. G., Sherwood, S. C., Siebesma, A. P., Sobel, A. H., Watanabe M., and Webb, M. J.: Clouds, circulation and climate sensitivity, Nat. Geosci., 8, 261-268, https://doi.org/10.1038/ngeo2398, 2015.

Canny, J.: A computational approach to edge-detection, IEEE T. Pattern Anal., 8, 679-698, 1986.

Clothiaux, E. E., Miller, M. A., Albrecht, B. A., Ackerman, T. P., Verlinde, J., Babb, D. M., Peters, R. M., and Syrett, W. J.: An evaluation of a 94-ghz radar for remote-sensing of cloud properties, J. Atmos. Ocean. Tech., 12, 201-229, https://doi.org/10.1175/15200426(1995)012<0201:aeoagr>2.0.co;2, 1995.

Clothiaux, E. E., Moran, K. P., Martner, B. E., Ackerman, T. P., Mace, G. G., Uttal, T., Mather, J. H., Widener, K. B., Miller, M. A., and Rodriguez, D. J.: The atmospheric radiation measurement program cloud radars: Operational modes, J. Atmos. Ocean. Tech., 16, 819-827, https://doi.org/10.1175/15200426(1999)016<0819:tarmpc>2.0.co;2, 1999.

Clothiaux, E. E., Ackerman, T. P., Mace, G. G., Moran, K. P., Marchand, R. T., Miller, M. A., and Martner, B. E.: Objective determination of cloud heights and radar reflectivities using a combination of active remote sensors at the ARM CART sites, J. Appl. Meteorol., 39, 645-665, https://doi.org/10.1175/15200450(2000)039<0645: odocha>2.0.co;2, 2000.

Fu, Q., Carlin, B., and Mace, G.: Cirrus horizontal inhomogeneity and OLR bias, Geophys. Res. Lett., 27, 3341-3344, https://doi.org/10.1029/2000gl011944, 2000a.
Fu, Q., Cribb, M. C., and Barker, H. W.: Cloud geometry effects on atmospheric solar absorption, J. Atmos. Sci., 57, 1156-1168, 2000 b.

Fu, Q., Baker, M., and Hartmann, D. L.: Tropical cirrus and water vapor: an effective Earth infrared iris feedback?, Atmos. Chem. Phys., 2, 31-37, https://doi.org/10.5194/acp-2-31-2002, 2002.

Fukao, S. and Hamazu, K.: Radar for Meteorological and Atmospheric Observations, Toyko, Springer, 2014.

He, K., Sun, J., and Tang, X.: Guided Image Filtering, IEEE T. Pattern Anal., 35, 1397-1409, https://doi.org/10.1109/tpami.2012.213, 2013.

Hildebrand, P. H. and Sekhon. R. S.: Objective Determination of the Noise Level in Doppler Spectra J. Appl. Meteorol., 13, 808-811, https://doi.org/10.1175/15200450(1974)013<0808:ODOTNL>2.0.CO;2, 1974.

Huang, J. P., Minnis, P., Lin, B., Yi, Y. H., Khaiyer, M. M., Arduini, R. F., Fan, A., and Mace, G. G.: Advanced retrievals of multilayered cloud properties using multispectral measurements, J. Geophys. Res.-Atmos., 110, D15S18, https://doi.org/10.1029/2004jd005101, 2005.

Huang, J. P., Minnis, P., Lin, B., Yi, Y. H., Fan, T. F., SunMack, S., and Ayers, J. K.: Determination of ice water path in ice-over-water cloud systems using combined MODIS and AMSR-E measurements, Geophys. Res. Lett., 33, L21801, https://doi.org/10.1029/2006gl027038, 2006a.

Huang, J. P., Wang, Y. J., Wang, T. H., and Yi, Y. H.: Dusty cloud radiative forcing derived from satellite data for middle latitude regions of East Asia, Prog. Nat. Sci., 16, 1084-1089, 2006 b.

Huang, J. P., Ge, J., and Weng, F.: Detection of Asia dust storms using multisensor satellite measurements, Remote Sens. Environ., 110, 186-191, https://doi.org/10.1016/j.rse.2007.02.022, 2007.

Huang, J., Zhang, W., Zuo, J., Bi, J., Shi, J., Wang, X., Chang, Z., Huang, Z., Yang, S., Zhang, B., Wang, G., Feng, G., Yuan, J., Zhang, L., Zuo, H., Wang, S., Fu, C., and Chou, J.: An Overview of the Semi-arid Climate and Environment Research Observatory over the Loess Plateau, Adv. Atmos. Sci., 25, 906-921, https://doi.org/10.1007/s00376-008-0906-7, 2008.

Illingworth, A. J., Hogan, R. J., O'Connor, E. J., Bouniol, D., Brooks, M. E., Delanoë, J., Donovan, D. P., Eastment, J. D., Gaussiat, N., Goddard, J. W. F., Haeffelin, M., Klein Baltink, H., Krasnov, O. A., Pelon, J., Piriou, J.-M., Protat, A., Russchenberg, H. W. J., Seifert, A., Tompkins, A. M., Van Zadelhoff, G.-J., Vinit, F., Willén, U., Wilson, D. R., and Wrench, C. L.: Cloudnet - Continuous evaluation of cloud profiles in seven operational models using ground-based observations, B. Am. Meteorol. Soc., 88, 883-898, https://doi.org/10.1175/bams-88-6-883, 2007.

Jing Su, Jianping Huang, Qiang Fu, Minnis, P., Jinming Ge, and Jianrong $\mathrm{Bi}$ : Estimation of Asian dust aerosol effect on cloud radiation forcing using Fu-Liou radiative model and CERES measurements, Atmos. Chem. Phys., 8, 2763-2771, https://doi.org/10.5194/acp-8-2763-2008, 2008.

King, M. D., Platnick, S., Menzel, W. P., Ackerman, S. A., and Hubanks, P. A.: Spatial and Temporal Distribution of Clouds Observed by MODIS Onboard the Terra and Aqua Satellites, IEEE T. Geosci. Remote, 51, 3826-3852, https://doi.org/10.1109/tgrs.2012.2227333, 2013.

Kollias, E., Clothiaux, E., Miller, M. A., Albrecht, B. A., Stephens, G. L., and Ackerman, T. P.: Millimeter-wavelength radars - New frontier in atmospheric cloud and precipitation research, B. Am. 
Meteorol. Soc., 88, 1608-1624, https://doi.org/10.1175/bams88-10-1608, 2007a.

Kollias, E., Clothiaux, E., Miller, M. A., Luke, E. P., Johnson, K. L., Moran, K. P., Widener, K. B., and Albrecht, B. A.: The Atmospheric Radiation Measurement Program cloud profiling radars: Second-generation sampling strategies, processing, and cloud data products, J. Atmos. Ocean. Tech., 24, 1199-1214, https://doi.org/10.1175/jtech2033.1, 2007b.

Li, J., Huang, J., Stamnes, K., Wang, T., Lv, Q., and Jin, H.: A global survey of cloud overlap based on CALIPSO and CloudSat measurements, Atmos. Chem. Phys., 15, 519-536, https://doi.org/10.5194/acp-15-519-2015, 2015.

Mace, G. G., Ackerman, T. P., Minnis, P., and Young, D. F.: Cirrus layer microphysical properties derived from surface-based millimeter radar and infrared interferometer data, J. Geophys. Res.Atmos., 103, 23207-23216, https://doi.org/10.1029/98jd02117, 1998.

Mace, G. G., Clothiaux, E. E., and Ackerman, T. P.: The composite characteristics of cirrus clouds: Bulk properties revealed by one year of continuous cloud radar data, J. Climate, 14, 2185-2203, https://doi.org/10.1175/15200442(2001)014<2185:tccocc $>2.0 . c 0 ; 2,2001$.

Marchand, R., Mace, G. G., Ackerman, T., and Stephens, G.: Hydrometeor detection using Cloudsat - An earth-orbiting 94-GHz cloud radar, J. Atmos. Ocean. Tech., 25, 519-533, https://doi.org/10.1175/2007jtecha1006.1, 2008.

Marr, D. and Hildreth, E.: Theory of edge-detection, P. R. Soc. B, 207, 187-217, https://doi.org/10.1098/rspb.1980.0020, 1980.

Perona, P. and Malik, J.: Scale-space and edge-detection using anisotropic diffusion, IEEE T. Pattern Anal., 12, 629-639, https://doi.org/10.1109/34.56205, 1990.

Protat, A., Bouniol, D., Delanoe, J., May, P. T., Plana-Fattori, A., Hasson, A., O'Connor, E., Goersdorf, U., and Heymsfield, A. J.: Assessment of Cloudsat Reflectivity Measurements and Ice Cloud Properties Using Ground-Based and Airborne Cloud Radar Observations, J. Atmos. Ocean. Tech., 26, 1717-1741, https://doi.org/10.1175/2009jtecha1246.1, 2009.

Protat, A., Delanoë, J., May, P. T., Haynes, J., Jakob, C., O'Connor, E., Pope, M., and Wheeler, M. C.: The variability of tropical ice cloud properties as a function of the large-scale context from ground-based radar-lidar observations over Darwin, Australia, Atmos. Chem. Phys., 11, 8363-8384, https://doi.org/10.5194/acp-11-8363-2011, 2011.

Ramanathan, V., Cess, R. D., Harrison, E. F., Minnis, P., Barkstrom, B. R., Ahmad, E., and Hartmann, D.: Cloud-radiative forcing and climate - Results from the earth radiation budget experiment, Science, 243, 57-63, https://doi.org/10.1126/science.243.4887.57, 1989.

Randall, D. A., Wood, R. A., Bony, S., Colman, R., Fichefet, T., Fyfe, J., Kattsov, V., Pitman, A., Shukla, J., Srinivasan, J., Stouffer, R. J., Sumi, A., and Taylor, K. E.: Cilmate Models and Their Evaluation, in: Climate Change 2007: The Physical Science Basis, Contribution of Working Group I to the Fourth Assessment Report of the Intergovernmental Panel on Climate Change, edited by: Solomon, S., Qin, D., Manning, M., Chen, Z., Marquis, M., Averyt, K. B., Tignor, M., and Miller, H. L., Cambridge University Press, Cambridge, United Kingdom and New York, NY, USA, 2007.
Sassen, K. and Benson, S.: A midlatitude cirrus cloud climatology from the facility for atmospheric remote sensing. Part II: Microphysical properties derived from lidar depolarization, J. Atmos. Sci., 58, 2103-2112, https://doi.org/10.1175/15200469(2001)058<2103:amcccf>2.0.co;2, 2001.

Stephens, G. L.: Cloud feedbacks in the climate system: A critical review, J. Climate, 18, 237-273, https://doi.org/10.1175/jcli3243.1, 2005.

Stephens, G. L., Vane, D. G., Boain, R. J., Mace, G. G., Sassen, K., Wang, Z., Illingworth, A. J., O'Connor, E. J., Rossow, W. B., Durden, S. L., Miller, S. D., Austin, R. T., Benedetti, A., Mitrescu, C., and The CloudSat Science Team: The cloudsat mission and the a-train - A new dimension of space-based observations of clouds and precipitation, B. Am. Meteorol. Soc., 83, 1771-1790, https://doi.org/10.1175/bams-83-12-1771, 2002.

Thorsen, T. J. and Fu, Q.: Automated Retrieval of Cloud and Aerosol Properties from the ARM Raman Lidar. Part II: Extinction, J. Atmos. Ocean. Tech., 32, 1999-2023, https://doi.org/10.1175/jtech-d-14-00178.1, 2015.

Thorsen, T. J., Fu, Q., and Comstock, J.: Comparison of the CALIPSO satellite and ground-based observations of cirrus clouds at the ARM TWP sites, J. Geophys. Res.-Atmos., 116, D21203, https://doi.org/10.1029/2011jd015970, 2011.

Thorsen, T. J., Fu, Q. N., Rob, K., Turner, D. D., Comstock, J. M.: Automated Retrieval of Cloud and Aerosol Properties from the ARM Raman Lidar. Part I: Feature Detection, J. Atmos. Ocean. Tech., 32, 1977-1998, https://doi.org/10.1175/JTECHD-14-00150.1, 2015.

Tomasi, C. and Manduchi, R.: Bilateral Filtering for Gray and Color Images, IEEE International Conference on Computer Vision, Bombay, India, https://doi.org/10.1109/ICCV.1998.710815, 1998.

Wang, Z. and Sassen, K.: Cloud type and macrophysical property retrieval using multiple remote sensors, J. Appl. Meteorol., 40, 1665-1682, https://doi.org/10.1175/15200450(2001)040<1665:ctampr>2.0.co;2 2001.

Williams, K. D. and Webb, M. J.: A quantitative performance assessment of cloud regimes in climate models, Clim. Dynam., 33, 141-157, https://doi.org/10.1007/s00382-008-0443-1, 2009.

Yan, H. R., Huang, J. P., Minnis, P., Yi, Y. H., Sun-Mack, S., Wang, T. H., and Nakajima, T. Y.: Comparison of CERESMODIS cloud microphysical properties with surface observations over Loess Plateau, J. Quant. Spectrosc. Ra., 153, 65-76, https://doi.org/10.1016/j.jqsrt.2014.09.009, 2015.

Yuan, J., Fu, Q., and McFarlane, N.: Tests and improvements of GCM cloud parameterizations using the CCCMA SCM with the SHEBA data set, Atmos. Res., 82, 222-238, https://doi.org/10.1016/j.atmosres.2005.10.009, 2006. 